\title{
L'émergence d'une migration féminine autonome du milieu rural vers le milieu urbain au Burkina FASO?
}

\author{
Gael Le Jeune \\ Etudiante au doctorat \\ Département de démographie \\ Université de Montréal
}

\author{
Jean Poirier \\ Coordonnateur du Centre interuniversitaire \\ québécois de statistiques sociales \\ Université de Montréal
}

\begin{abstract}
Résumé
Dans cet article, nous utilisons les données de l'enquête nationale "Dynamique migratoire, insertion urbaine et environnement au Burkina Faso - 2000" pour vérifier l'hypothèse de l'émergence d'une migration féminine autonome du milieu rural vers le milieu urbain au Burkina Faso. La migration "autonome" est définie comme une migration visant à satisfaire des objectifs économiques individuels. Nous montrons que la migration autonome n'explique que partiellement la recrudescence des migrations de femmes célibataires du milieu rural vers le milieu urbain. Les migrations de femmes mariées, qui restent prépondérantes entre le milieu rural et le milieu urbain au Burkina Faso, répondent essentiellement, quant à elles, à des impératifs familiaux. Cependant, on observe que le mariage avec un migrant peut, dans certains cas, répondre à des objectifs économiques individuels. Finalement, les changements relevés apparaissent assez subtiles et s'inscrivent plutôt dans la continuité.
\end{abstract}

\section{Introduction}

Selon des travaux récents, on assisterait aujourd'hui à un développement sans précédent des migrations autonomes des femmes en Afrique de l'ouest (Findley, 1997 ; Antoine et Sow, 2000 ; Bocquier et Traoré, 2000 ; Adepoju, 2002). La migration "autonome" des femmes est définie dans ces travaux comme une migration visant à satisfaire les besoins économiques individuels des femmes. Elle résulterait principalement de la "féminisation de la pauvreté" qui affecterait particulièrement les milieux ruraux de la région (Adepoju, 2002). Mais le phénomène n'est peut-être pas nouveau. En fait, on ne dispose tout simplement pas des données qui nous permettraient d'apprécier l'évolution du comportement migratoire des femmes sur de longues périodes.

Le Burkina Faso fait, à ce titre, figure d'exception. En 1974-75, une vaste enquête rétrospective menée auprès d'un échantillon national stratifié de plus de 93000 individus donnait déjà des informations inédites sur le comportement migratoire des femmes dans les trois premiers quarts du $20^{\text {ième }}$ siècle (Cordell et al., 1996). En 2000, une nouvelle enquête nationale rétrospective complétait le tableau en retraçant cette fois non seulement les itinéraires migratoires des individus mais aussi leur trajectoire scolaire, professionnelle et familiale depuis l'âge de six ans (Poirier et al., 2001).

Dans cet article, nous utiliserons les données de cette dernière enquête pour vérifier l'hypothèse de l'émergence d'une migration autonome des femmes entre le milieu rural et le milieu urbain au Burkina Faso dans le dernier quart du $20^{\text {ième }}$ siècle. 
Nous commencerons par rappeler succinctement quel a été l'apport de la théorie à la compréhension du comportement migratoire des femmes et nous poursuivrons par une présentation du contexte burkinabé.

\section{Revue de littérature}

\section{Les femmes dans la théorie migratoire}

Comme le remarquent Pessar et Malher (2001), les rapports de genre ont jusqu'à nos jours façonné tant les migrations que la recherche sur les migrations. En fait, le comportement migratoire des femmes, qui a été longtemps ignoré puis simplifié, remet largement en question la pertinence des modèles explicatifs conventionnels et de nombreux auteurs plaident pour la construction de nouveaux schémas centrés sur la notion de genre (Grieco et Boyd, 1998 ; Hondagneu-Sotelo et Cranford, 1999 ; Kanaiaupuni, 2000 ; Pessar et Malher, 2001) :

"Besides neglecting and stereotyping migrant women, migration research often uses conceptual models that are gender insensitive and thus fail to fully explain or emphasize the interactions between gender and migration.", Grieco et Boyd (1998 : 2).

La théorie migratoire reste avant tout marquée par l'apport des économistes néoclassiques qui, tout en plaçant la liberté des individus au centre de leurs modèles, ont considéré les femmes comme des êtres totalement passifs. Pour Sjaastad (1962), par exemple, les individus se déplacent de manière à maximiser leurs revenus en fonction de leur capital humain mais, pas les femmes, qui semblent être par nature des "dépendants"1. Certains économistes ont tenté de remédier à cette aberration dont Mincer (1978) qui a notamment lancé le concept de "tied move". En fait, les gains que le conjoint peut espérer en migrant contrebalancent les pertes éventuellement subies par la femme qui l'accompagne et il en résulte un gain net pour toute la famille. Dès lors, les femmes choisissent librement de suivre leur conjoint. Mais dans la mesure où les activités féminines ont tendance à être systématiquement dévalorisées, la rationalité économique des individus qui fonde ce type de modèles a été largement remise en question (Lawson, 1998 ; Halfacree et Boyle, 1999).

D'autres économistes néoclassiques, qui se sont intéressés aux migrations dans les pays en développement, n'ont pas tenu compte du sexe des migrants (Harris et Todaro, 1970) ou encore ont construit des modèles spécifiquement féminins (Thadani et Todaro, 1984). Dans ce dernier modèle, les femmes migrent en fonction des opportunités matrimoniales qui s'offrent à elles à défaut d'opportunités d'emploi. Cependant, Behrman et Wolfe (1984), qui ont utilisé le modèle de Thadani et Todaro dans le contexte du Nicaragua, ont montré qu'en dépit de leur position marginale sur le marché $\mathrm{du}$ travail urbain, les migrantes restent principalement motivées par les opportunités d'emploi qui s'offrent à elles en ville. 
Contrairement à l'approche néoclassique qui se focalise sur l'individu, l'approche structuraliste se concentre, comme son nom l'indique, sur les structures qui, au niveau macro, conditionnent les comportements individuels. Cette approche a surtout été utilisée pour expliquer les migrations de travail en mettant en évidence le rôle des inégalités de développement. On reproche généralement à ce type de travaux de négliger les relations entre la sphère capitaliste et la sphère domestique dans laquelle évoluent notamment les femmes (Grieco et Boyd, 1998). Le ménage se trouve être le "point d'articulation" entre ces deux sphères (Saint-Pierre et al. 1986). En conséquence, c'est à ce niveau que se décideraient les migrations des individus qui constitueraient un moyen parmi d'autres pour le ménage de se perpétuer compte tenu des contraintes imposées par son environnement physique et socio-économique (Wood, 1982).

Que la migration représente pour le ménage, notamment dans les pays en développement, une stratégie de survie (Wood, 1982) ou de diversification des risques (Stark, 1991), le processus de décision à l'intérieur du ménage demeure obscur. En fait, le concept de "stratégie des ménages" a été vivement critiqué car il suppose l'existence d'un consensus entre les membres du ménage (Folbre, 1987 ; Crow, 1989 ; Wolf, 1990 ; Moen et Wethington, 1992). Or, les stratégies des uns peuvent entrer en conflit avec les stratégies des autres. Il arrive notamment que le chef de ménage exerce un pouvoir despotique provocant des comportements qui ne s'inscrivent pas dans une stratégie proprement dite mais qui reflètent plutôt des tentatives de résistance désordonnées (Riley et Gardner, 1993).

Il n'en reste pas moins que les hiérarchies internes aux ménages entre les sexes et les générations expliquent en grande partie les profils migratoires des hommes et des femmes (Lawson, 1998 ; Hondagneu-Sotelo et Cranford, 1999). Cette approche qui se fonde véritablement sur la notion de genre ${ }^{2}$ a été développée par des auteurs féministes et utilisée notamment en Amérique latine :
"Research in a variety of settings has examined cultural constructions of women's and men's roles finding that women's positions within household divisions of labour produce very different reasons for, and patterns of, mobility.", Lawson (1998: 42).

Dans cette perspective, la mobilité d'une femme dépend avant tout de sa position au sein de son ménage ainsi que de la localisation des différents membres, le ménage étant défini comme un ensemble de personnes (le plus souvent apparentées) mettant leurs ressources, entièrement, ou partiellement en commun mais ne vivant pas forcement sous le même toit (Lawson, 1998). Finalement, la famille qui se confond avec le ménage ainsi défini et qui joue un tout premier rôle dans l'institution de rôles et de statuts très différenciés entre les individus apparaît comme le contexte dans lequel les migrations féminines doivent d'abord être étudiées (Lim, 1995 ; Grieco et Boyd, 1998). 


\section{Le contexte burkinabé}

Le Burkina Faso est un pays essentiellement rural - le taux d'urbanisation ne s'élève qu'à 16\% selon les chiffres du dernier recensement de 1996 (INSD, 2000a). La majorité de la population y pratique une agriculture de subsistance à forte intensité de main-d'œuvre dans un environnement difficile compte tenu des aléas climatiques et de la pauvreté des sols (INSD, $2000 b)$. La production s'organise sur une base familiale selon des règles très strictes. Ouédraogo (2000:3) résume ainsi l'organisation de la société chez les Mossi $^{3}$ en milieu rural :

"[...] ceux sont les aînés du lignage qui exercent le contrôle sur la circulation des femmes, qui contrôlent la production et qui assurent l'autorité. La filiation et patrilinéaire et la résidence virilocale. Cette organisation consacre la domination de l'homme sur les femmes et les enfants; elle est donc de type très inégalitaire. Les normes légitiment ces rapports inégaux qui se traduisent par des rôles très sexués."

Ainsi, la famille est de type patriarcale et gérontocratique. Du fait rôle primordial que les femmes jouent dans la reproduction de la force de travail, leurs déplacements sont sévèrement contrôlés.

En fait, les aînés assoient une grande partie de leur pouvoir sur leur capacité à donner des femmes en mariage à leurs cadets (Capron et Kohler, 1978 ; Gruénais, 1985). En conséquence, les migrations féminines sont avant tout le produit de contrats matrimoniaux qui peuvent avoir été négociés sans l'accord des femmes concernées. On s'attend à ce que les femmes respectent le choix de conjoint qui a été fait pour elles en rejoignant le domicile que ce dernier leur assigne. Même si, dans les faits, les jeunes femmes participent de plus en plus activement aux processus matrimoniaux (Capron et Kohler, 1978 ; Laurent, 1996), il n'en reste pas moins que les femmes mariées se placent sous l'autorité de leur conjoint et n'ont pas en principe le loisir de se déplacer librement. Le mariage est précoce et universel : plus de la moitié des femmes ont déjà contracté une union à 18 ans et la quasi-totalité à 25 ans (INSD et Macro, 2000). Entre 25 et 45 ans, les femmes qui ne sont pas en union représentent moins de $5 \%$ des femmes : en cas de rupture d'union, le remariage est très rapide.

Par ailleurs, les devoirs et obligations des femmes mariées sont bien souvent incompatibles avec leurs déplacements. En effet, les femmes mariées sont responsables de l'entretien du ménage au quotidien 4 et, à moins d'avoir une coépouse, elles peuvent difficilement s'absenter (Findley, 1997). Elles assurent également une grande partie du travail agricole (Gueilla et Poirier, 2000 ; Kevane et Wydick, 2001). La femme idéale est donc celle qui est "présente dans la maison" (Ouédroago, 2000). Le statut social des femmes mariées s'améliore néanmoins avec l'âge. Et l'arrivée d'une belle-fille dans le ménage les libère complètement de toutes leurs obligations productives et reproductives (Gruénais, 1985). 
En définitive, les "migrations de mariage" et les "visites" (forme de migration temporaire à caractère social) sont les deux formes autorisées de la mobilité féminine au Burkina Faso. Dans un tel contexte, il paraît difficile d'imaginer l'émergence d'une "migration autonome" des femmes qui est pourtant annoncée dans la littérature.

\section{L'émergence d'une migration féminine autonome?}

Bien que les données sur la migration, en général, et sur la migration féminine, en particulier, restent parcellaires ou inadéquates en Afrique subsaharienne (Oucho, 1998), de nombreux auteurs évoquent une intensification de l'exode rural des femmes et parallèlement une intensification des migrations féminines qui peuvent être qualifiées d'"autonomes" notamment en Afrique de l'ouest (Findley, 1997 ; Antoine et Sow, 2000 ; Bocquier et Traoré, 2000 ; Adepoju, 2002) :

"Mais les migrations féminines ne sont-elles pas le fruit de mutations plus profondes des sociétés ouestafricaines, à mettre en rapport avec l'amélioration de l'éducation des filles? Ne correspondent-elles pas à une aspiration de plus en plus grande des femmes à l'autonomie, notamment par le travail ?", Bocquier et Traoré $(2000: 84)$.

De nombreuses études de cas semblent par ailleurs conforter l'hypothèse de l'émergence d'une migration féminine autonome du milieu rural vers le milieu urbain dans divers pays d'Afrique de l'ouest en particulier chez les jeunes femmes célibataires (Brydon, 1992 ; Delauney, 1994 ; Olurode, 1995 ; Ouédraogo, 1995 ; Hertrich et Lesglingand, 2001) ${ }^{5}$.

Au Burkina Faso, l'exode rural est essentiellement dirigé vers les pays côtiers limitrophes, notamment la Côte-d'Ivoire, où la culture intensive de produits d'exportation comme le café nécessite une main-d'œuvre importante. Un système de migrations circulaires de travail s'est par ailleurs développé tout au long du 20ième siècle entre le Burkina et la Côte-d'Ivoire (Cordell et al., 1996). Mais jusqu'en 1975, les femmes participaient peu à ce courant migratoire : en 1969-73, on comptait quatre migrations masculines pour une migration féminine à destination de l'étranger (Coulibaly et al., 1980). Cependant, dans les trois premiers quarts du 20 ième siècle, on a pu observer une multiplication des migrations féminines à destination de l'étranger, d'une part, et du milieu urbain burkinabé, d'autre part (Cordell et al., 1996). Cette tendance semble s'être poursuivie depuis (Le Jeune et al., à paraître). Les femmes sont de plus en plus nombreuses à quitter le milieu rural et, bien que l'étranger constitue toujours la principale destination, son attrait semble diminuer au profit du milieu urbain burkinabé notamment dans les générations les plus jeunes. Les célibataires représentent une proportion croissante de ces migrantes. Elles viennent en ville pour y poursuivre des études, chercher ou prendre un travail ou encore pour des motifs familiaux divers. Cependant, la majorité des femmes qui migrent du milieu rural vers le milieu urbain sont encore des femmes mariées qui rejoignent ou accompagnent leur conjoint. 


\section{Hypothèses et variables retenues}

Dans cet article, nous nous proposons de vérifier, avec des données longitudinales, l'hypothèse de l'émergence d'une migration féminine autonome du milieu rural vers le milieu urbain au Burkina Faso. Pour mettre en oeuvre le concept d'autonomie, nous postulons que le capital humain accumulé par les femmes au niveau individuel (niveau d'éducation, expérience professionnelle et migratoire) a une influence prépondérante sur leur propension à migrer de manière autonome alors que, à l'inverse, les migrations des femmes que l'on peut qualifier de "dépendantes" sont plutôt déterminées par leur situation familiale. Nous distinguons deux catégories de femmes, les célibataires et les mariées, dont les situations familiales ne sont pas comparables et qui, par ailleurs, constituent l'essentiel du flux migratoire entre le milieu rural et le milieu urbain.

Les variables de capital humain constituent les variables indépendantes principales. On tiendra compte non seulement du niveau d'éducation mais aussi de l'expérience professionnelle et migratoire des femmes. Le capital humain est donc mesuré en nombre d'années accumulées de scolarité et de travail rémunéré et, plus particulièrement pour les femmes mariées, en nombre d'années de résidence à l'extérieur du milieu rural, en milieu urbain et à l'étranger, accumulées avant le dernier épisode d'union.

On s'intéresse en outre au statut dans l'activité du moment qui pourrait être plus déterminant que les qualifications et l'expérience accumulée par les femmes. On distingue les femmes actives occupées non rémunérées, c'est-àdire les aides familiales, les actives occupées rémunérées (indépendantes pour la plupart), les inactives scolarisées (il s'agit des femmes en cours de scolarisation), les chômeuses et les inactives non scolarisées. Les épisodes de chômage étant extrêmement rares chez les femmes en milieu rural, nous regroupons ces deux dernières catégories.

Les variables qui rendent compte de la situation familiale des femmes constituent les variables de contrôle. Nous avons relevé dans la littérature sur les migrations en Afrique une série de variables qui pourraient être pertinentes.

Ainsi, le nombre de frères et sœurs du côté du père et de la mère nous donne la taille approximative de la famille d'origine. On s'attend à ce que les individus qui appartiennent à de grandes familles migrent plus facilement que les autres : d'une part, la famille sera moins déstabilisée par la perte d'un de ses membres ; d'autre part, le migrant aura plus de chances de retrouver un membre de sa famille à la destination (Findley, 1997). Mais, selon Caldwell (1968), l'effet de cette variable devrait être contrôlé par le rang de naissance qui aurait un effet négatif : les cadets étant appelés à soutenir leurs parents, ils auraient moins la possibilité de migrer. 
La catégorie socioprofessionnelle des parents nous renseigne sur les origines sociales des femmes ainsi que sur les activités de la famille d'origine. Aux deux extrémités de l'échelle sociale, nous distinguons, d'un côté, les agriculteurs et éleveurs, de l'autre, l'ensemble des cadres moyens ou supérieurs des secteurs privé et public, les professions libérales, les employés de la fonction publique et des forces de l'ordre.

Le décès des parents peut grandement affecter la trajectoire des femmes. Les données disponibles (qui spécifient la date de décès des parents) montrent que ce facteur varie dans le temps. On peut penser que les orphelines bénéficient de moins de soutien au sein des ménages, ce qui peut compromettre un projet migratoire ou au contraire l'encourager et ce d'autant plus que les parents biologiques ne sont plus là pour exercer leur autorité.

Le statut de résidence nous donne, pour chaque épisode de résidence, le lien entre la femme et le chef de ménage. En effet, les femmes peuvent être hébergées par leurs parents, par leurs beaux-parents ou par d'autres personnes. Les femmes qui se déclarent locataires ou propriétaires peuvent l'être à leur propre chef ou à celui de leur conjoint quand elles sont mariées. On peut s'attendre à ce que les femmes qui sont en situation périphérique dans un ménage soient plus mobiles que les autres.

Nous avons construit un indice de confort dans le logement à partir des caractéristiques de l'habitat pour chaque épisode de résidence. Il traduit le niveau d'aisance du ménage. Les femmes qui vivent dans des ménages pauvres peuvent être incitées à migrer. Cependant, la migration a un coût, aussi les migrants appartiendraient plutôt à des ménages relativement aisés (Byerlee, 1974).

Le nombre d'enfants à charge, c'est-à-dire que le nombre d'enfants que la femme a mis au monde et qui ne sont ni décédés ni partis, peut influencer la mobilité des femmes. On s'attend également à ce que les femmes qui allaitent aient une moindre propension à migrer car la migration présente alors un risque pour leur propre santé et celle de l'enfant. De plus, l'enfant peut difficilement être confié (Brockerhoff et $\mathrm{Eu}, 1993$ ). Par ailleurs la présence d'enfants plus âgés peut inciter les femmes à migrer pour bénéficier des services de santé et d'éducation dont l'offre est plus abondante en ville. La présence d'enfants plus âgés peut également, à l'inverse, inciter les femmes à rester dans la mesure où elles bénéficient de l'aide de ces enfants et leur statut social est renforcé en milieu rural (Brockerhoff et Eu, 1993). Aussi, nous distinguerons, parmi les enfants qui sont encore à charge, les enfants de moins de 6 mois (ces enfants sont encore allaités), les enfants de 6 mois à 6 ans et les enfants de 7 ans ou plus, scolarisés et non scolarisés.

Enfin, nous avons pu accoler les biographies des conjoints aux biographies des femmes mariées au moment de l'enquête quand ces derniers ont été interrogés, ce qui représente $75 \%$ des cas 6 . Pour ces femmes et pour toute la 
durée de leur dernière union, on dispose donc, à chaque instant, du milieu de résidence du conjoint. Cette variable devrait en grande partie expliquer le comportement migratoire des femmes mariées si on admet qu'elles sont dépendantes et ne font que suivre leur conjoint. Nous avons également tenu compte du statut matrimonial du conjoint (monogame ou polygame) qui varie dans le temps. Les femmes qui ont au moins une coépouse devrait être plus mobiles que les autres (Findley, 1997) comme nous l'avons déjà évoqué.

\section{Données et méthodologie}

Nous disposons de données particulièrement adaptées pour vérifier l'hypothèse d'une recrudescence des migrations féminines à caractère autonome entre le milieu rural et le milieu urbain au Burkina Faso. En effet, en 2000, plus de 8600 biographies d'hommes et de femmes ont été collectées au Burkina Faso auprès d'un échantillon national stratifié représentatif de la population résidante âgée de 15 à 64 ans lors de la première phase de collecte de l'enquête "Dynamique migratoire, insertion urbaine et environnement au Burkina Faso". L'objectif principal de cette enquête était de permettre une meilleure compréhension des "stratégies de reproduction" des populations du Burkina Faso en vue d'éclairer la prise de décision (Poirier et al., 2001).

Les biographies qui ont alors été recueillies sont multidimensionnelles : elles contiennent des informations sur l'ensemble des épisodes de résidence, d'activité et d'union des individus depuis l'âge de six ans. Pour les femmes, ces données comportent en outre l'histoire de chacun de leur enfant né vivant. Nous pouvons donc retracer l'enchaînement des événements qui ont marqué la vie des femmes et déceler des relations de cause à effet au niveau individuel. Mais nous avons aussi pu partiellement reconstituer leur environnement familial à partir de quelques variables spécifiques.

Nous chercherons à expliquer les migrations définies comme des changements de résidence pour une durée de un an ou plus entraînant un changement de milieu de résidence du rural vers l'urbain. Le milieu urbain est défini comme l'ensemble des localités de 10000 habitants ou plus. Il s'est continuellement agrandi au cours de la période que nous étudierons (Le Jeune et al., à paraître). La durée minimum de un an de résidence à destination a été retenue pour minimiser les biais de mémoire. En effet, les femmes les plus âgées ont des difficultés à se rappeler des épisodes de résidence de courtes durées intervenus dans leur jeunesse contrairement aux femmes les plus jeunes (Smith et Duncan, 1997).

Avant de procéder à des régressions multi-variées, nous présenterons quelques indicateurs sur l'évolution du phénomène. Nous calculerons d'abord des taux de migrations par état matrimonial et par période pour mettre en évidence l'intensification des migrations féminines du milieu rural vers le milieu urbain au Burkina Faso au cours des 25 dernières années et 
souligner l'importance respective des migrations de femmes célibataires et mariées. Puis nous construirons des courbes de séjour en milieu rural à partir de 10 ans pour les femmes célibataires ou de l'entrée en union pour les femmes mariées et nous vérifierons que les femmes appartenant aux cohortes les jeunes ont des comportements significativement différents des femmes appartenant aux cohortes plus âgés.

Enfin, nous nous livrerons à une analyse explicative proprement dite. Nous effectuerons des régressions sur la propension des femmes célibataires ou mariées à quitter le milieu rural. Nous utiliserons le modèle semiparamétrique à risques proportionnel de Cox (1972) en introduisant dans le modèle, outre la cohorte de naissance, des variables explicatives individuelles et familiales.

Le modèle de Cox est un modèle de régression particulier qui tient compte des durées de séjour des individus dans les différents états que l'on a identifiés comme pouvant exercer une influence sur le risque de connaître l'événement étudié. On peut écrire que :

$$
\log [r(t)]=a(t)+\sum_{i} b_{i} X_{i}+\sum_{j} c_{j} y_{j}(t)
$$

où $r(t)$ représente le risque de migrer à l'instant $t$ $a(t)$ est une fonction non spécifiée du temps $x_{i}$ sont les variables explicatives fixes $y_{j}(t)$ sont les variables explicatives qui varient dans le temps

L'ensemble des coefficients bi et cj sont estimés par rapport à des modalités de référence selon la méthode de la "vraisemblance partielle".

Avant de procéder à des régressions pour mettre en évidence lesquelles des variables de capital humain ou de situation familiale expliquent le mieux la propension à migrer des femmes célibataires et mariées, nous allons montrer que les migrations du milieu rural vers le milieu urbain sont de plus en plus le fait de femmes célibataires au Burkina Faso.

\section{Résultats}

\section{L'essor des migrations de célibataires}

Nous avons comptabilisé toutes les migrations du milieu rural vers le milieu urbain intervenues entre 15 et 35 ans dans 8 cohortes de femmes d'origine rurale $^{7}$ selon la période, le groupe d'âges et le statut matrimonial de ces femmes au moment de la migration. En rapportant ces nombres de migrations aux effectifs des cohortes concernées, on obtient des taux de migrations par période, groupe quinquennaux d'âges et statut matrimonial. Nous avons pondéré ces taux afin de tenir compte de la structure par âge de la population féminine en milieu rural et nous les avons additionnés pour obtenir des taux standardisés de migration par statut matrimonial et par période (Figure 1). 


\begin{tabular}{|c|r|r|r|r|r|}
\hline \multirow{2}{*}{$\begin{array}{c}\text { Statut matrimonial au } \\
\text { moment de la migration }\end{array}$} & \multicolumn{5}{|c|}{ Périodes } \\
\cline { 2 - 6 } & $1975-79$ & $1980-84$ & $1985-89$ & $1990-94$ & $1995-99$ \\
\hline Célibataires & $0,4 \%$ & $0,2 \%$ & $0,4 \%$ & $0,6 \%$ & $0,7 \%$ \\
\hline Mariées & $1,4 \%$ & $2,0 \%$ & $2,0 \%$ & $4,0 \%$ & $3,0 \%$ \\
\hline Veuves ou divorcées & $0,1 \%$ & $0,1 \%$ & $0,0 \%$ & $0,0 \%$ & $0,4 \%$ \\
\hline Total & $1,9 \%$ & $2,3 \%$ & $2,5 \%$ & $4,6 \%$ & $4,1 \%$ \\
Effectifs & 1057 & 1291 & 1459 & 1629 & 1556
\end{tabular}

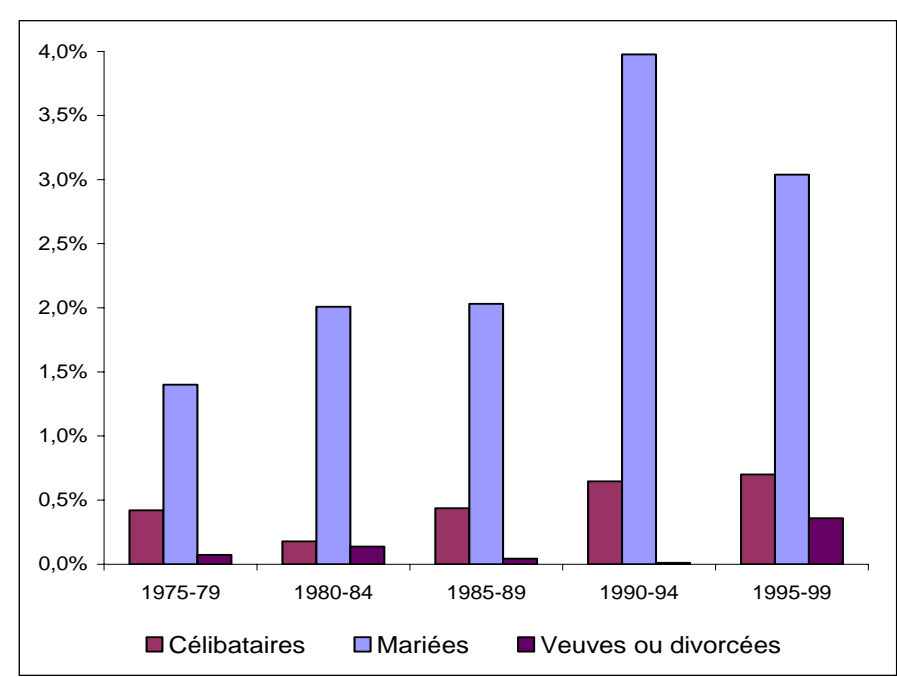

Source : Données biographiques de l'enquête "Dynamique migratoire, insertion urbain et environnement au Burkina Faso - 2000".

Figure 1 : Taux de migration du milieu rural vers le milieu urbain des femmes entre 15 et 35 ans selon le statut matrimonial et la période

Si on examine ces derniers taux, on constate que l'intensité de la migration féminine a tendance à augmenter de période en période : le taux de migration du milieu rural vers le milieu urbain (tous statuts matrimoniaux confondus) passe de 1,9\% en 1975-79 à 4,1\% en 1995-99. L'intensification du phénomène est assez nette au début des années 1980 et surtout au début des années 1990. Mais on remarque que ce taux de migration baisse à la fin des années 1990. Un certain essoufflement de la croissance urbaine enregistré dans l'ensemble des pays de la région (Bocquier et Traoré, 2000) affecterait ainsi la propension des femmes à migrer du milieu rural vers le milieu urbain dans la dernière période.

Si on examine les taux de migration par statut matrimonial, on voit que seul le taux de migration de femmes mariées diminue au cours de la période 1995-99 tandis que le taux de migration de femmes célibataires poursuit sa 
progression. Cependant, les célibataires ne sont responsables que de $17 \%$ des migrations des femmes d'origine rurale entre 15 et 35 ans dans cette dernière période ( $c^{\prime}$ est moins qu'en 1975-79 où la proportion des migrations de célibataires atteignait $22 \%$ ). Les migrations de femmes en rupture d'union (veuves ou divorcées) restent, quant à elles, relativement marginales.

Les courbes de séjour que l'on peut construire nous montrent que les femmes d'origine rurale appartenant aux générations les plus jeunes semblent avoir plus de chances de migrer à destination du milieu urbain que les femmes appartenant aux générations les plus âgées mais ce résultat n'est significatif que chez les célibataires.

Dans l'ensemble de l'échantillon, on compte en effet 2955 femmes célibataires et non-sorties du milieu rural à 10 ans. Sur ces 2955 femmes, 223 ont effectué une première sortie du milieu rural à destination du milieu urbain en tant que célibataires avant l'enquête. En tenant compte des pondérations, des troncatures que constituent les trajectoires interrompues par l'enquête ainsi que des événements perturbateurs que constituent les mariages et/ou les premières sorties du milieu rural à destination de l'étranger, on peut estimer des courbes de séjour en l'état de célibataires non-sorties du milieu rural à partir de 10 ans (Figure 2). On constate immédiatement que les femmes appartenant à la cohorte la plus jeune (197185) semblent avoir eu nettement plus de chances de migrer à destination du milieu urbain que les femmes appartenant à la cohorte la plus âgée (19361970). Cependant, l'intensité du phénomène reste très faible : en définitive, $9 \%$ des femmes migrent à destination du milieu urbain en tant que célibataire dans la cohorte la plus jeune contre 3\% dans la cohorte la plus âgée.

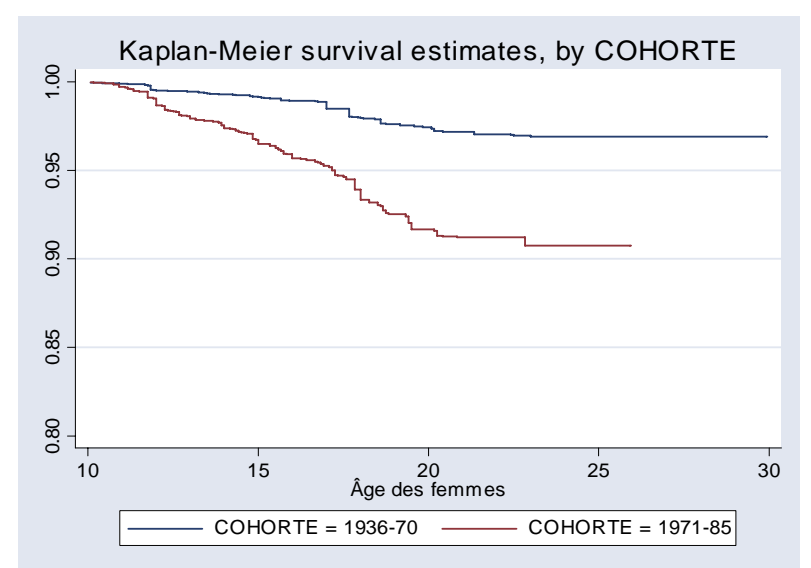

Source : Données biographiques de l'enquête "Dynamique migratoire, insertion urbaine et environnement au Burkina Faso - 2000".

Figure 2 : Proportion de femmes célibataires qui n'ont pas encore effectué de migration du milieu rural vers le milieu urbain selon l'âge et la cohorte 
Sur les 2955 femmes célibataires et non-sorties du milieu rural à 10 ans, 1733 sont entrées dans un dernier épisode d'union en milieu rural ${ }^{8}$, cet épisode s'est prolongé jusqu'à la date de l'enquête et nous avons pu retrouver la biographie du conjoint (ce qui nous permettra de construire des variables explicatives spécifiques ultérieurement). Sur ces 1733 femmes, 317 ont effectué une première sortie du milieu rural à destination du milieu urbain au cours de ce dernier épisode d'union. En fait, nous considérons que les femmes sont mariées un mois avant le début d'un épisode de cohabitation ${ }^{9}$. En tenant compte des pondérations, des troncatures et des événements perturbateurs (sorties du milieu rural à destination de l'étranger), on peut estimer des courbes de séjour en l'état de mariées nonsorties du milieu rural à partir de l'entrée dans le dernier épisode d'union (Figure 3). Ces courbes ont une allure particulière du fait de l'importance des migrations de mariage : environ la moitié des femmes mariées appelées à migrer le font dans le premier mois du mariage. L'intensité du phénomène migratoire est légèrement plus forte chez les femmes mariées que chez les femmes célibataires : $12 \%$ des femmes mariées migrent à destination du milieu urbain dans la cohorte le plus jeune contre $7 \%$ dans la cohorte la plus âgée. Les femmes mariées nées en 1971-85 semblent donc avoir eu plus de chances de migrer à destination du milieu urbain que les femmes mariées nées en 1936-70. Mais chez les femmes mariées, contrairement à ce qu'on observait chez les célibataires, les cohortes n'ont pas de comportements significativement différents comme nous le verrons bientôt.

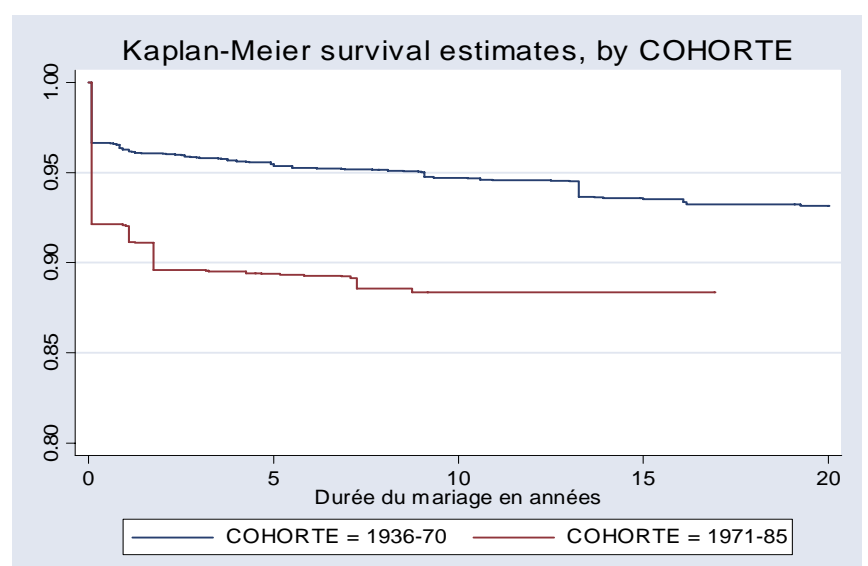

Source : Données biographiques de l'enquête "Dynamique migratoire, insertion urbaine et environnement au Burkina Faso - 2000".

Figure 3 : Proportion de femmes mariées qui n'ont pas encore effectué de migration du milieu rural vers le milieu urbain selon la durée du mariage et la cohorte

\section{Des femmes célibataires en quête d'autonomie?}

En introduisant uniquement la cohorte comme variable explicative dans notre modèle de régression (Tableau 1 , colonne $\mathrm{A}$ ), on voit que, chez les 
femmes célibataires, il existe bien une différence significative de comportement entre les femmes nées en 1971-85 et les femmes nées en 193670. L'introduction de l'ensemble de nos variables explicatives dans le modèle réduit très peu cet effet de cohorte (Tableau 1, colonne B) ce qui signifie que les femmes célibataires nées après 1970 ont une plus forte propension à migrer à destination du milieu urbain pour des raisons qui ne sont pas entièrement saisies par les variables de notre modèle. On peut penser qu'il s'agit là d'un effet d'entraînement : les migrantes célibataires qui rentrent au village sont fréquemment dépeintes comme suscitant l'envie auprès de leurs congénères du fait des bijoux, des habits et de la vaisselle ${ }^{10} q^{\prime}$ 'elles ont $\mathrm{pu}$ acquérir en ville (Delauney, 1994 ; Compaoré et Ouattara, 1999 ; Hertrich et Lesglingand, 2001). Aussi, les migrations circulaires de femmes célibataires se développent et sont de mieux en mieux encadrées et acceptées.

S'agissant des variables de capital humain, on voit que plus les femmes sont éduquées et plus elles ont de chances de migrer à destination du milieu urbain. L'expérience professionnelle mesurée par le nombre d'années d'activité rémunérée n'a pas d'effet significatif. En revanche, la situation dans l'activité du moment est particulièrement déterminante. Les actives occupées rémunérées, c'est-à-dire essentiellement les femmes qui mènent une activité en tant qu'indépendante en milieu rural, ont significativement plus de chances de migrer que les aides familiales. Les inactives scolarisées ou non et les chômeuses ont également significativement plus de chances de migrer mais dans une moindre mesure. Le comportement des célibataires semble donc se conformer à la théorie du capital humain : les variables individuelles telles que le niveau d'éducation et le fait de mener une activité indépendante jouent de façon décisive.

Quant aux variables familiales, en dehors de la catégorie socioprofessionnelle $\mathrm{du}$ père et $\mathrm{du}$ décès de la mère, aucune variable n'a d'effet significatif sur la propension des femmes célibataires à migrer à destination du milieu urbain. La catégorie socioprofessionnelle du père a un effet significatif dans le sens attendu : quand le père a (ou a eu s'il est décédé) pour activité principale une autre activité que celle d'agriculteur ou éleveur, en particulier quand le père appartient aux catégories intermédiaires ou supérieures (cadres moyens ou supérieurs dans les secteurs privé ou public, les professions libérales, les employés de la fonction publique et des forces de l'ordre), les femmes migrent plus facilement à destination du milieu urbain. On peut penser que ces femmes effectuent des migrations d'accompagnement de leur père ou encore que ce type de père encourage la migration de leurs filles à destination du milieu urbain. Le décès de la mère, en revanche, réduit significativement les chances des femmes célibataires de migrer à destination du milieu urbain. On peut penser que la mère constitue un soutien important à la migration des célibataires ou encore que les filles sont appelées à se substituer à leur mère décédée au niveau du travail domestique et sur l'exploitation familiale. 
http://aps.journals.ac.za

114 African Population Studies Vol.20 n²/Etude de la population africaine vol. $20 n^{\circ} 2$

Tableau 1 : Coefficients estimés pour la première migration à destination du milieu urbain des femmes célibataires

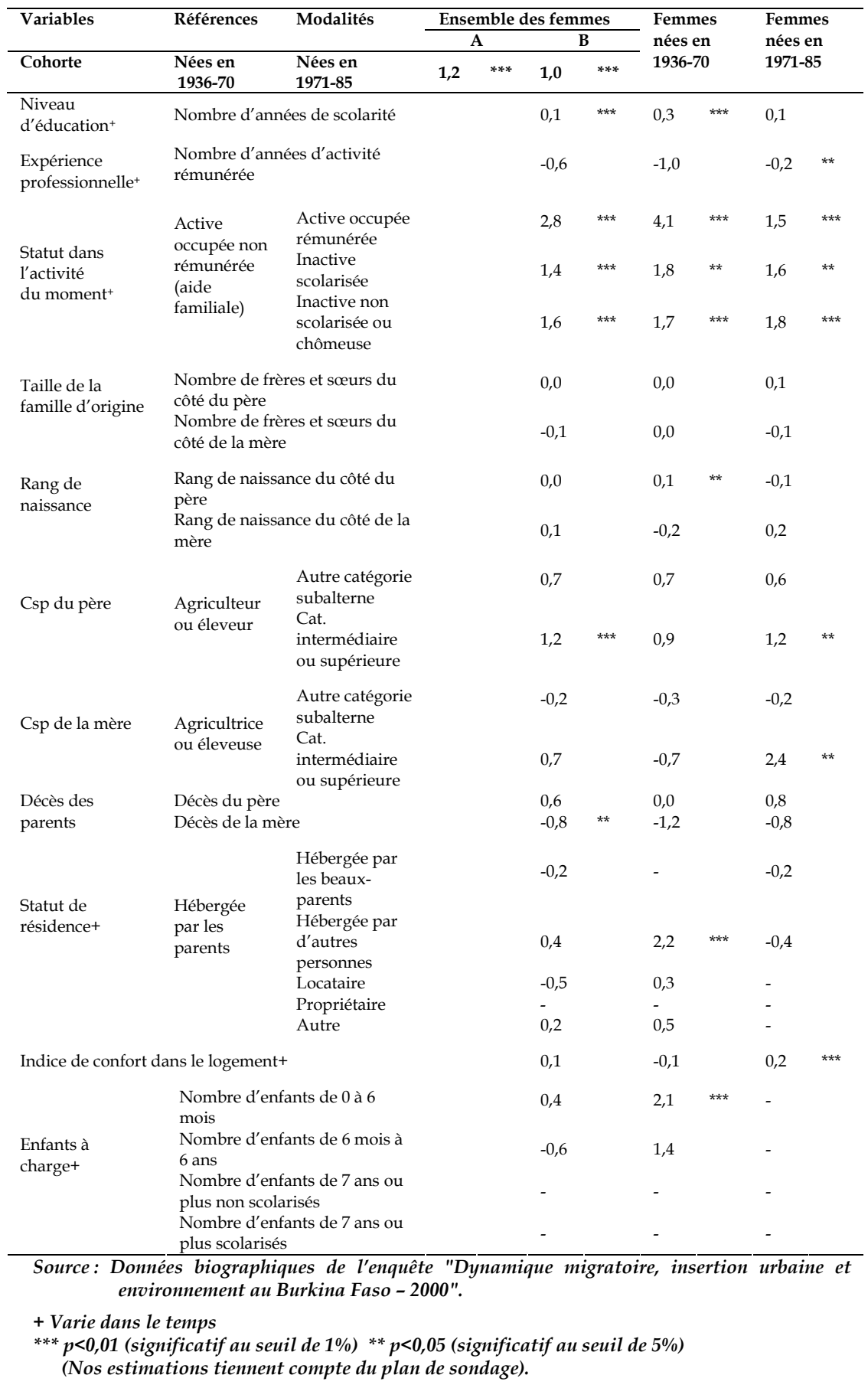


Chez les femmes nées en 1936-70, les variables de capital humain jouent beaucoup plus fortement que chez les femmes nées en 1971-85. Chez les premières, on constate en outre que les célibataires qui sont des cadettes, qui sont hébergées par d'autres personnes que leurs parents biologiques et qui sont à charge d'enfants âgés de 6 mois à 6 ans ont significativement plus de chances de migrer que les autres. Chez les femmes nées en 1971-85, ce sont les célibataires dont le père et surtout la mère appartiennent aux catégories socioprofessionnelles intermédiaires ou supérieures et qui résident dans des ménages relativement aisés qui ont significativement plus de chances de migrer à destination du milieu urbain.

Paradoxalement, chez les femmes nées en 1971-85, l'expérience professionnelle mesurée en nombre d'années d'activité rémunérée accumulées a un effet négatif significatif sur la propension à migrer à destination du milieu urbain alors que le fait d'être active rémunérée a un effet positif significatif. En fait, on peut penser que l'expérience professionnelle acquise par les femmes qui commencent très tôt à travailler comme indépendante (ces femmes ont très fréquemment une mère également indépendante dans les secteurs de la production et de la vente de produits alimentaires ou de l'artisanat) est relativement bien valorisée en milieu rural et elle les dissuadent de migrer.

De cohorte en cohorte, on voit donc que les déterminants de la migration des célibataires évoluent. Chez les femmes les plus jeunes, le niveau de vie du ménage et le type d'activité exercée par les parents semblent prendre de l'importance au détriment des caractéristiques individuelles des femmes. On peut penser que les migrations d'accompagnement sont plus fréquentes chez ces femmes ou encore que la famille s'investit plus dans leurs migrations.

\section{Des femmes mariées qui ne se contentent pas de suivre leur conjoint}

Comme nous le mentionnions précédemment, il n'y a pas de différence significative entre le comportement des femmes mariées nées en 1936-70 et celui des femmes mariées nées en 1971-85 (Tableau 2, colonne A).

À l'introduction de l'ensemble de nos variables explicatives (Tableau 2, colonne B), on remarque immédiatement que les variables de capital humain n'ont pratiquement pas d'effet significatif sur la propension des femmes mariées à migrer à destination du milieu urbain contrairement à ce qu'on observait chez les célibataires. On constate néanmoins que les inactives non scolarisées et les chômeuses ont significativement plus de chances de migrer que les autres femmes. Autrement dit, les femmes mariées qui ont une occupation en milieu rural, c'est-à-dire essentiellement les indépendantes et les aides familiales, sont plus disposées à rester en milieu rural, quelle que soit la trajectoire migratoire de leur conjoint. 
Comme on pouvait s'y attendre, le fait que le conjoint réside déjà en milieu urbain incite très fortement les femmes mariées à migrer. On note que les femmes rejoignent plus volontiers leur conjoint quand ce dernier réside à Ouagadougou ou Bobo Dioulasso que dans une autre ville.

Certaines caractéristiques de la famille d'origine sont également déterminantes, ce qui n'est pas surprenant étant donné la définition du mariage que nous avons retenue : nous avons considéré que les femmes sont mariées un mois avant le début d'un épisode de cohabitation, ce qui implique qu'une bonne partie des femmes résident encore chez leurs parents alors qu'elles entrent en observation. On constate que le décès de la mère favorise la migration des femmes mariées contrairement à ce qu'on observait chez les célibataires. On peut penser que ces femmes se marient plus facilement que les autres avec des migrants. Le fait d'avoir (ou d'avoir eu si elle est décédée) une mère appartenant aux catégories socioprofessionnelles subalternes autres que celles d'agricultrice ou d'éleveuse (il s'agit essentiellement de femmes actives dans les secteurs de la production et la vente de produits alimentaires et de l'artisanat) favorise également la migration des femmes mariées à destination du milieu urbain.

Les femmes mariées ont bien souvent des enfants en charge, ce qui peut influencer leur mobilité. Il apparaît que la présence d'enfants d'âge préscolaire mais qui ont dépassé l'âge critique de 6 mois encourage les femmes à migrer alors que la présence d'enfants de 7 ans ou plus les dissuade plutôt de migrer en particulier quand ils ne sont pas scolarisés.

Enfin, on note que les femmes mariées qui sont propriétaires, c'est-à-dire, pour la plupart, dont le mari est propriétaire en milieu rural, ont une moindre propension à migrer à destination du milieu urbain, ce qui est assez compréhensible.

Quand on s'intéresse aux différentes cohortes de femmes mariées, on constate que le fait d'être inoccupée en milieu rural a surtout un impact sur la propension à migrer des femmes nées après 1970 . Chez ces femmes, on remarque également que le fait d'avoir déjà séjourné en milieu urbain (avant le dernier épisode d'union) a un effet positif significatif sur leur propension à migrer à nouveau à destination du milieu urbain. Chez les femmes nées en 1936-70, au contraire, seul le fait d'être en cours de scolarisation a un impact positif significatif au niveau des variables de capital humain.

Au niveau des variables qui caractérisent la situation familiale des femmes, on note que les femmes appartenant aux générations les plus jeunes ont une propension nettement plus élevée que les femmes appartenant aux générations plus âgées à rejoindre leur conjoint dès lors que ce dernier réside en milieu urbain. Le comportement migratoire des femmes mariées apparaît donc comme de plus en plus dépendant de celui de leur conjoint ce qui peut sembler contraire à l'hypothèse d'une plus grande autonomie des femmes. En fait, comme le remarque Traoré (2000), il est probable que ce résultat reflète un renforcement de l'autorité du conjoint au détriment de celle des beaux-parents. 
http://aps.journals.ac.za

G. Le Jeune, V. Piché E J. Poirier : L'émergence d'une migration féminine autonome

Tableau 2 : Coefficients estimés pour la première migration à destination du milieu urbain des femmes mariées

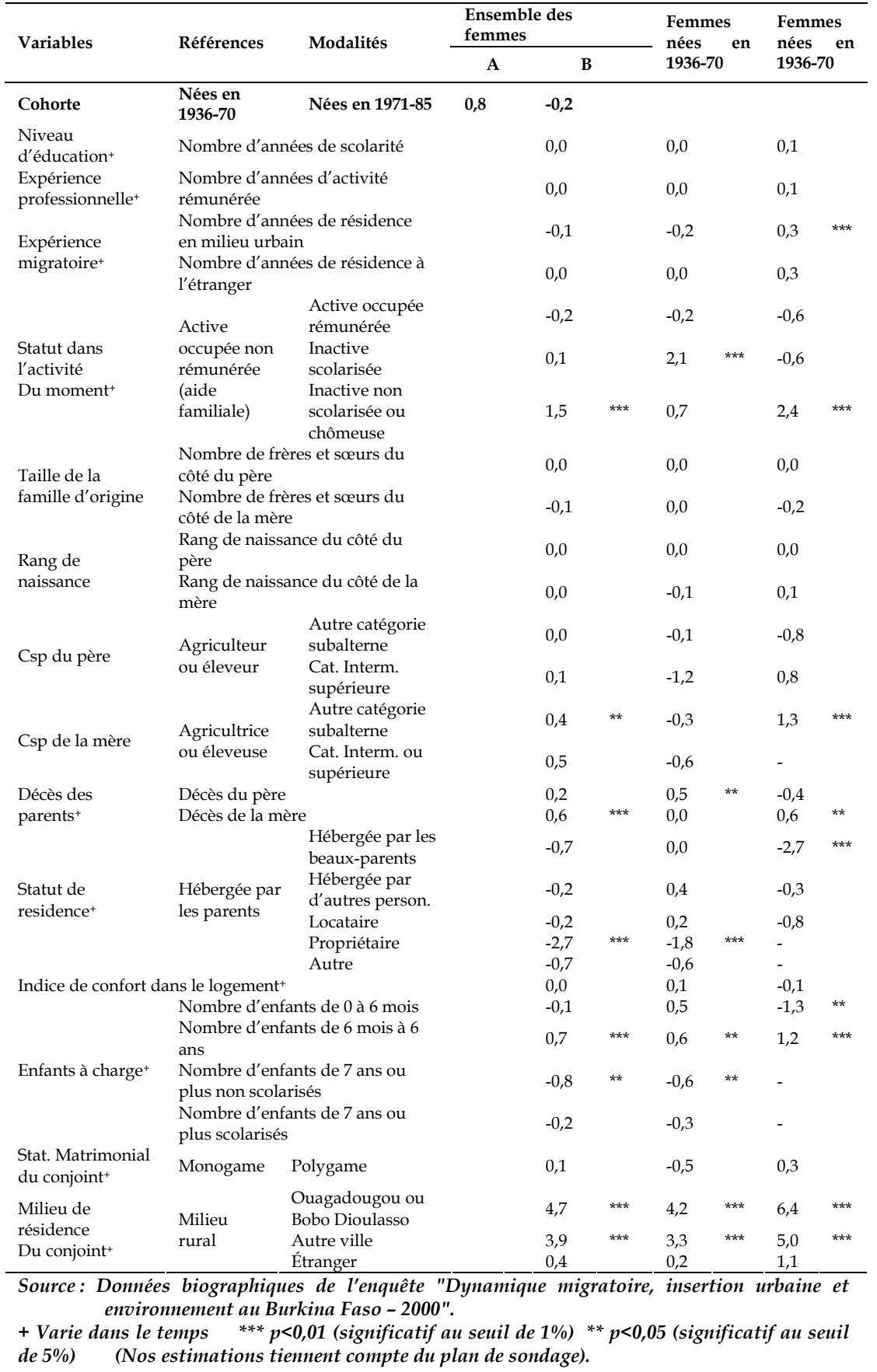


http://aps.journals.ac.za

118 African Population Studies Vol. $20 n^{\circ} 2 /$ Etude de la population africaine vol. $20 n^{\circ} 2$

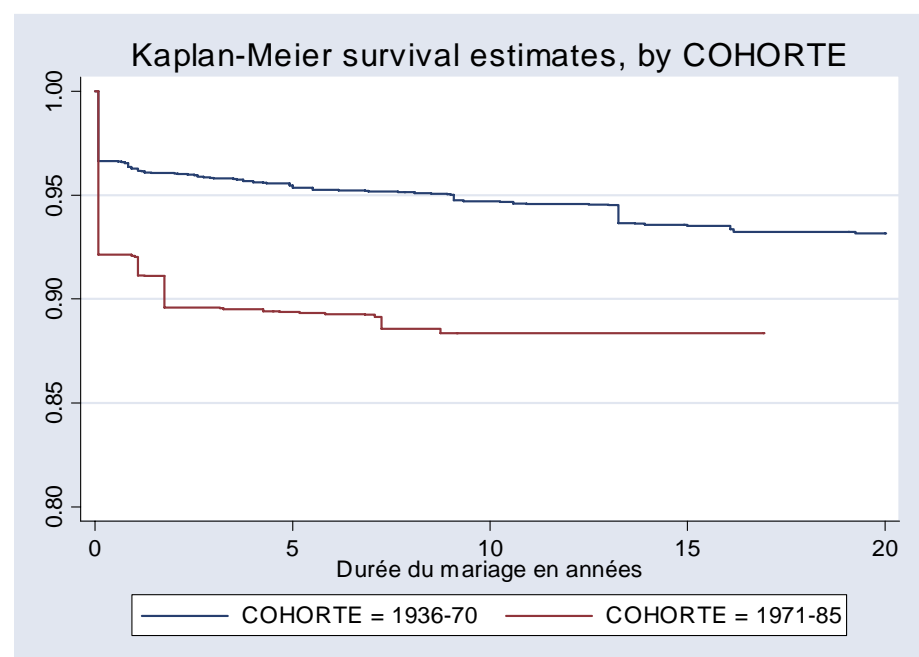

Source: Données biographiques de l'enquête "Dynamique migratoire, insertion urbaine et environnement au Burkina Faso - 2000".

Figure 4 : Proportion de femmes mariées qui n'ont pas encore effectué de migration du milieu rural vers le milieu urbain selon la durée du mariage et la cohorte

\section{Discussion}

Au Burkina Faso, les trois quarts des femmes qui migrent du milieu rural vers le milieu urbain sont des femmes mariées qui rejoignent leur conjoint à destination. Le comportement migratoire des femmes semble donc largement induit par le comportement migratoire des hommes. Cependant, nos résultats montrent que non seulement les migrations de célibataires sont en augmentation mais que le mariage avec un migrant pourrait bien constituer un moyen alternatif pour des femmes en quête d'autonomie d'échapper à leur milieu d'origine.

Concernant les migrations féminines, Cordell, Gregory et Piché (1996 : 231) avaient intitulé un chapitre de leur ouvrage sur les migrations au Burkina Faso dans les trois premiers quarts du 20ième siècle: "The men followed the money and the women followed the men". Ils concluaient que les migrations féminines n'avaient pas fondamentalement changé de nature au terme de cette période : les femmes continuaient de circuler principalement à l'intérieur de la "sphère domestique". Notre analyse de la migration féminine du milieu rural vers le milieu urbain au Burkina Faso pour le dernier quart du $20^{\text {ième }}$ siècle ne met pas non plus en lumière de véritable révolution dans le comportement des femmes. Nous avons plutôt mis en évidence des changements dans la continuité.

D'une part, les migrations de femmes célibataires restent très minoritaires bien qu'elles prennent de l'importance. Et, dans les générations les plus 
jeunes, les femmes résidant en milieu rural ont toujours plus de chances de migrer à destination du milieu urbain dans le cadre du mariage qu'en tant que célibataires. La migration des célibataires semble répondre à des motivations économiques individuelles. Cependant, on constate que les caractéristiques individuelles des célibataires comme leur niveau d'éducation ou leur situation dans l'activité du moment ont de moins en moins d'importance face à certains paramètres familiaux comme la catégorie socioprofessionnelle des parents ou le niveau de vie du ménage. En fait, les emplois précaires occupés par les femmes à destination - notamment depuis le début de la "crise" qui sévit en milieu urbain - leur permettent très difficilement d'acquérir une véritable indépendance financière et la moitié des migrantes arrivées célibataires en milieu urbain sont appelées à effectuer une migration de retour vers le milieu rural (résultats qui ne sont pas présentés ici). Dans ces conditions, l'essor des migrations de femmes célibataires du milieu rural vers le milieu urbain ne doit pas être trop rapidement interprété comme un mouvement de libération des femmes. Les épisodes de résidence en milieu urbain représentent néanmoins pour les femmes d'origine rurale des occasions de s'affirmer en tant qu'individu et ils pourraient à terme modifier les rapports de genre en milieu rural (Hertrich et Lesglingand, 2001).

D'autre part, on observe que le comportement migratoire des femmes mariées est relativement peu sensible à leurs caractéristiques individuelles. En revanche, le milieu de résidence du conjoint est pour ces femmes déterminant (variable qui n'apparaissait bien évidemment pas dans les facteurs explicatifs de la migration des femmes célibataires). Les femmes nées après 1970 se distinguent. Elles ont, en effet, une plus forte propension à migrer à destination du milieu urbain dès lors qu'elles sont sans emploi en milieu rural ou qu'elles ont déjà séjourné en milieu urbain. Elles ont également une plus forte propension à suivre leur conjoint en milieu urbain dès lors que ce dernier s'y installe comparativement aux femmes appartenant aux générations plus âgées. Ainsi, dans un contexte où la mobilité des femmes est particulièrement restreinte et l'autonomie par le travail difficilement réalisable, le mariage apparaît comme une structure d'opportunité qui peut être exploitée par les femmes qui voudraient quitter définitivement leur milieu d'origine.

En définitive, les femmes en quête d'autonomie ont pu emprunter des chemins multiples et la période récente ne semble pas radicalement différente des précédentes au Burkina Faso. La dynamique de l'insertion des migrantes sur le marché du travail urbain mériterait à ce stade d'être éclairer afin d'évaluer dans quelle mesure la migration peut répondre aux aspirations des femmes d'origine rurale. 


\section{Notes}

1. Sjaastad oppose le migrant, qualifié de "human agent", et sa famille, ses "dependents".

2. "The term 'gender' rather than 'sex' is deliberate. 'Gender' explicitely rejects biological explanations for hierarchies of inequality and power that privilege men and disadvantage women. Rather than being a fixed trait, invariant over time and space, gender denotes the social construction of identities, behavior and power relationships (Scott, 1986).", Grieco et Boyd (1998).

3. Dans les autres groupes ethniques, la situation des femmes diffère peu.

4. Les femmes doivent notamment : chercher de l'eau, apporter ou produire les condiments, préparer le repas, donner à manger et à boire au mari, aux enfants, aux beaux-parents, assurer la propreté du logement, faire la toilette des enfants, les surveiller (Ouédraogo, 2000).

5. Findley (1989) et Antoine et Sow (2000) proposent des revues de ces études.

6. $25 \%$ des conjoints n'ont pas été interrogés pour les principales raisons suivantes : ils résidaient ailleurs au moment de l'enquête, ils étaient absents, trop vieux pour répondre, ils ont refusé.

7. En fait, tout au long de cet article, nous ne nous intéresserons qu'aux femmes qui ont commencé leur trajectoire migratoire en milieu rural à six ans et qui n'étaient toujours pas sorties du milieu rural à 10 ans. Ces femmes ont été socialisées en milieu rural. Nous dirons qu'elles sont d'"origine rurale".

8. Une partie de ces femmes a séjourné en milieu urbain ou à l'étranger entre temps.

9. "La cohabitation existe juridiquement dès l'instant où le mari a affecté une résidence à la femme", Adjamagbo-Johnson (1997 : 250). Nos données comportent non seulement la date de début de cohabitation mais aussi les dates des cérémonies coutumière, religieuse et civile qui ont éventuellement été célébrées pour chaque épisode d'union.

10. Ces objets font partie du trousseau de mariage.

\section{Références}

Adepoju, A. 2002. "Fostering Free Movement of Persons in West Africa: Achievements, Constraints, and Prospects for Intraregional Migration." International Migration 40, (2): 3-28.

Adjamagbo-Johnson, B. 1997, "Législations et changements familiaux en Afrique subsaharienne francophone." Pp. 240-256. In Ménages et familles en Afrique: approches des dynamiques contemporaines, Edited by M. Pilon, T. Locoh, K. Vignikin and P. Vimard. Paris: CEPED.

Antoine, P. and Sow O., 2000. "Rapports de genre et dynamiques migratoires: le cas de l'Afrique de l'ouest." Pp. 143-159. In Rapports de genre et questions de population. II - Genre, population et développement: les pays du Sud , Edited by M. Bozon and T. Locoh. Paris: INED.

Behrman, J.R. and Wolfe, B.L. 1984. "Micro determinants of female migration in a developing country: labor market, demographic marriage market and economic marriage market incentives." Research in population economics 5: 137-166. 
Bocquier, P. and Traoré, S. 2000. Urbanisation et dynamique migratoire en Afrique de l'ouest: la croissance urbaine en panne. Paris: L'Harmattan.

Brockerhoff, M. and Eu, H. 1993. "Demographic and Socioeconomic Determinants of Female Rural to Urban Migration in Sub-Saharan Africa." International Migration Review 27, (3): 557-577.

Brydon, L. 1992. "Ghanaian Women in the Migration Process." Pp. 91-108. In Gender and Migration in Developing Countries, Edited by S. CHANT. London and New York: Belhaven Press.

Byerlee, D. 1974. "Rural-urban Migration in Africa: Theory, Policy, and Research Implications." International Migration Review 8, (4): 543-566.

Caldwell, J. 1968. "Determinants of Rural-Urban Migration in Ghana." Population Studies 22, (3): 361-77.

Capron, J. and Kohler, J.-M. 1978. "De quelques caractéristiques de la pratique matrimoniale Mossi contemporaine." Pp. 187-223. In Marriage, Fertility and Parenthood in West Africa, Edited by C. Oppong, G. Adaba, M. Bekombo-Priso and J. Mogey. Canberra: Department of Demography, Australian National University.

Compaoré, M. and Ouattara, A. 1999. "Mobilité féminine et insertion urbaine des migrantes à Ouagadougou, Burkina Faso." Paper presented for: "Être étranger et migrant en Afrique au XXème siècle. Modes d'insertion et enjeux identitaires". Conférence. Paris. 9-11 december.

Cordell, D.; Gregory, J.W. and Piché, V. 1996. Hoe \& Wage. A Social History of a Circular Migration System in West Africa. Boulder: Westview Press.

Coulibaly, S.; Gregory, J.W. and Piché, V. 1980. Les migrations voltaïques, Tome I: Importance et ambivalence de la migration voltaïque. Ouagadougou: Centre voltaïque de la recherche scientifique and INSD and Ottawa: CRDI.

Cox, D.R. 1972. "Regression Models and Life-Tables." Journal of the Royal Statistical Society, Series B (Methodological) 34: 187-220.

Crow, G. 1989. "The Use of the Concept of 'Strategy' in Recent Sociological Litterature." Sociology 23, (1): 1-24.

Delaunay, V. 1994. L'entrée en vie féconde: expression démographique des mutations socio-économiques d'un milieu rural sénégalais. Paris: CEPED.

Findley, S.E. 1989. "Les migrations féminines dans les villes africaines: leurs motivations et experiences." Pp. 55-70. In L'insertion urbaine des migrants en Afrique, Edited by P. Antoine and S. Coulibaly. Paris: ORSTOM.

Findley, S.E. 1997. Migration and Family Interactions in Africa Pp. 109-138. In Family, Population and Development in Africa, Edited by A. ADEPOJU. London: Zed Books.

Folbre, N. 1987. "Family Strategy, Feminist Strategy." Historical Methods 20, (3): 115118.

Grieco, E.M. and Boyd, M. 1998. "Women and Migration: Incorporating Gender Into International Migration Theory.". Florida State University Center for the Study of Population Working Paper 139.

Gruénais, M.-É. 1985. "Aînés, aînées ; cadets, cadettes. Les relations aînés/cadets chez les Mossi du centre (Burkina Faso)." Pp. 219-246. In Âge, pouvoir et société en Afrique noire, Edited by M. Abélès and C. Colard. Paris: Karthala.

Guiella, G. and Poirier, J. 2000. "Fondements socio-économiques de la fécondité chez les mossi du plateau central (Burkina Faso)." Pp. 93-110. In Maîtrise de la fécondité et planification familiale au Sud, Edited by M. Pilon and A. Guillaume. Paris: IRD.

Halfacree, K. and Boyle, P. 1999. "Introduction. Gender and Migration in Developed Countries." Pp. 1-29. In Migration and Gender in the Developed World, Edited by P. Boyle and K. Halfacree. London and New York: Routledge. 
Harris, J.R. and Todaro, M.P. 1970. "Migration, Unemployment and Development: A Two Sector Analysis." American Economic Review 60, (1): 126142.

Hertrich, V. and Lesclingand, M. 2001. "Entrée dans l'âge adulte en milieu rural africaIn vers une convergence des trajectoires masculines et féminines ? Le cas des Bwa du Mali." Paper presented for: XXIV UIESP General Congress. Salvador. 18-24 August.

Hondagneu-Sotelo, P. and Cranford, C. 1999. "Gender and Migration." Pp. 105-127. In Handbook of the Sociology of Gender, Edited by J.S. CHAFETZ. New York: Kluwer Academic/Plenum Publishers.

INSD and MACRO INTERNATIONAL, 2000. Enquête démographique et de santé, Burkina Faso, 1998-1999. Ouagadougou: Institut National de la Statistique et de la Démographie.

INSD, 2000a. Analyse des résultats du recensement général de la population et de l'habitation de 1996, Volume I. Ouagadougou: Ministère de l'Économie et des Finances and Institut national de la statistique et de la démographie.

INSD, 2000b. Profil et évolution de la pauvreté au Burkina Faso. Ouagadougou: Ministère de l'économie et des finances and Institut national de la statistique et de la démographie, Direction des statistiques générales.

Kanaiaupuni, S.M. 2000. "Refraiming the Migration Question: An Analysis of Men, Women, and Gender in Mexico." Social Forces 78:1311-1348.

Kevane, M. and Wydick, B. 2001. "Social norms and the time allocation of women's labor in Burkina Faso." Review of Development Economics 5, (1): 119-129.

Laurent, P.-J. 1996. "Dynamiques matrimoniales chez les Mossi (Burkina Faso). Pratiques de l'Église des Assemblées de Dieu, des aînés et de l'État." Cahiers africains 19-20: 166-183.

Lawson, V. 1998. "Hierarchical Households and Gendered Migration in Latin America: Feminist Extensions to Migration Research." Progress in Human Geography 22, (1): 39-53.

Le Jeune, G.; Piché, V. and Poirier, J. à paraître. "Female Migration Patterns in Burkina Faso : A Changing Configuration." Canadian Studies in Population.

Lim, L.L. 1995. "The Status of Women and International Migration." Pp. 29-55. In International Migration Policies and the Status of Female Migrants. Proceedings of the United Nations Expert Group Meeting in International Migration Policies and the Status of Female Migrants. San Miniato. 28-31 March 1990. New York: United Nations.

Mincer, J. 1978. "Family Migration Decisions." Journal of Political Economy 86, (5): 749-773.

Moen, P. and Wethington, E. 1992. "The Concept of Family Adaptative Strategies." Annual Review of Sociology 18: 233-51.

Olurode, L. 1995. "Women in Rural-Urban Migrations in the Town of Iwo in Nigeria." Pp. 289-302. In The Migration Experience in Africa, Edited by J. BAKER and T.A. AINA. Uppsala: Nordiska Afrikainstitutet.

Oucho, J.O. 1998. "Recent Internal Migration Processes in Sub-Saharan Africa: Determinants, Consequences, and Data Adequacy Issues." Pp. 89-120. In Migration, Urbanization, and Development: New Directions and Issues, Edited by R.E. BILSBORROW. New York: UNFPA.

Ouédraogo, C. 2000. "Femmes africaines: à la recherche de leur statut." Paper presented for: Population et développement. Le développement peut-il être humain et durable ? Chaire Quételet. Louvain-la-Neuve. 21-24 November.

Ouédraogo, J.-B. 1995. "The Girls of Nyovuuru. Dagara Female Labour Migrations to Bobo-Dioulasso." Pp. 303-320. In The migration experience in Africa, Edited by J. BAKER and T.A. AINA. Uppsala: Nordiska Afrikainstitutet. 
Pessar, P.R. and Mahler, S.J. 2001. "Gender and Transnational Migration." Princeton University Working paper 06e.

Poirier, J.; Piché, V.; Le Jeune, G.; Dabiré, B. and Wane, H.R. 2001. "Projet d'étude des stratégies de reproduction des populations sahéliennes à partir de l'enquête 'Dynamique migratoire, insertion urbaine et environnement au Burkina Faso'." Cahiers québécois de démographie 30, (2): 289-310.

Riley, N.E. and Gardner, R.W. 1993. "Migration Decisions: The Role of Gender." Pp. 195-206. In Internal Migration of Women in Developing Countries. Proceedings of the United Nations Expert Meeting on the Feminization of Internal Migration. Aguascalientes. 22-25 October 1991. New York: United Nations.

Saint-Pierre, M.-H.; Gregory, J.W. and Simmons, A.B. 1986. "Structure démographique des ménages et comportement migratoire en Haute-Volta (Burkina Faso)." Pp. 111-142. In Démographie et sous-développement dans le Tiers-Monde, Edited by D. Gauvreau, J.W. Gregory, M. Kempeneers and V. Piché. Montréal: Center for Developing-Area Studies.

Scott, J.W. 1986. "Gender: A Useful Category of Historical Analysis." American Historical Review 91, (5): 1053-75.

Sjasstad, L.A. 1962. "The Costs and Returns of Human Migration." Journal of Political Economy 70, (2): 80-93.

Smith, J.P. and Duncan, T. 1997. "Migration in Retrospect: Remembrances of Things Past." RAND Labor and Population Program Working Papers DRU-1628-NICHD.

Stark, O. 1991. The Migration of Labor. Cambridge and Oxford: B. Blackwell.

Thadani, V.N. and Todaro, M.P. 1984. "Female Migration: A Conceptual Framework." Pp. 36-59. In Women in the Cities of Asia: Migration and Urban Adaptation, Edited by J.T. Fawcett, S.-E. Khoo and P.C. Smith. Boulder: Westview Press.

Traoré, S. 2000. Migration et insertion socio-économique dans les villes en Afrique de l'ouest. Bamako: CERPOD-CILSS-INSAH.

Wolf, D.L. 1990. "Daughters, Decisions and Domination: An Empirical and Conceptual Critique of Household Strategies." Development and Change 21, (1): 43-74.

Wood, C.H. 1982. "Equilibrium and Historical-Structural Perspectives on Migration." Internation Migration Review 16, (2): 298-319. 